\title{
Determination of $f_{+}^{K}(0)$ and extraction of $\left|V_{c s}\right|$ from semileptonic $D$ decays
}

\author{
Y. Fang ${ }^{a}$, G. Rong, H. L. Ma, J. Y. Zhao \\ Institute of High Energy Physics, Beijing 100039, People's Republic of China
}

Received: 29 September 2014 / Accepted: 11 December 2014 / Published online: 14 January 2015

(C) The Author(s) 2014. This article is published with open access at Springerlink.com

\begin{abstract}
By globally analyzing all existing measured branching fractions and partial rates in different four momentum transfer-squared $q^{2}$ bins of $D \rightarrow K e^{+} v_{e}$ decays, we obtain the product of the form factor and magnitude of Cabibbo-Kobayashi-Maskawa matrix element $V_{c s}$ to be $f_{+}^{K}(0)\left|V_{c s}\right|=0.717 \pm 0.004$. With this product, we determine the $D \rightarrow K$ semileptonic form factor $f_{+}^{K}(0)=$ $0.737 \pm 0.004 \pm 0.000$ in conjunction with the value of $\left|V_{c s}\right|$ determined from the standard model global fit. Alternately, with the product together with the input of the form factor $f_{+}^{K}(0)$ calculated in lattice quantum chromodynamics (LQCD) recently, we extract $\left|V_{c s}\right|^{D \rightarrow K e^{+} v_{e}}=$ $0.962 \pm 0.005 \pm 0.014$, where the error is still dominated by the uncertainty of the form factor calculated in LQCD. Combining the $\left|V_{c s}\right|_{s}^{D_{s}^{+} \rightarrow \ell^{+} \nu_{\ell}}=1.012 \pm 0.015 \pm 0.009$ extracted from all existing measurements of $D_{s}^{+} \rightarrow \ell^{+} \nu_{\ell}$ decays and $\left|V_{c s}\right|^{D \rightarrow K e^{+} v_{e}}=0.962 \pm 0.005 \pm 0.014$ together, we find the most precisely determined $\left|V_{c s}\right|$ to be $\left|V_{c s}\right|=$ $0.983 \pm 0.011$, which improves the accuracy of the PDG'2014 value $\left|V_{c S}\right|^{\mathrm{PDG}^{\prime} 2014}=0.986 \pm 0.016$ by $45 \%$.
\end{abstract}

\section{Introduction}

In the standard model (SM) of particle physics, the mixing between the quark flavours in weak interaction is parameterized by the Cabibbo-Kobayashi-Maskawa (CKM) matrix, which is a $3 \times 3$ unitary matrix. Since the CKM matrix elements are fundamental parameters of the SM, precise determinations of these elements are necessary and very important in testing the SM and searching for new physics (NP).

Since the effects of strong interactions and weak interaction can be well separated in semileptonic $D$ decays, these decays are excellent processes from which we can determine the magnitude of CKM matrix element $V_{c s(d)}$.

\footnotetext{
a e-mail: fangy@ihep.ac.cn
}

In the SM, neglecting the lepton mass, the tree-level differential decay rate in absence of radiative correction for $D \rightarrow K e^{+} v_{e}$ process is given by

$\frac{d \Gamma}{d q^{2}}=\frac{G_{F}^{2}}{24 \pi^{3}}\left|V_{c s}\right|^{2} \boldsymbol{p}^{3}\left|f_{+}^{K}\left(q^{2}\right)\right|^{2}$,

where $G_{F}$ is the Fermi constant, $\boldsymbol{p}$ is the three momentum of the $K$ meson in the rest frame of the $D$ meson, $q^{2}$ is the four momentum transfer-squared, i.e. the invariant mass of the lepton and neutrino system, and $f_{+}^{K}\left(q^{2}\right)$ is the form factor which parameterizes the effect of strong interaction.

In addition to extraction of $\left|V_{c s}\right|$, the precise measurements of the $D \rightarrow K$ semileptonic form factor is also very important to validate the lattice quantum chromodynamics (LQCD) calculation of the form factor. If the LQCD calculation of the form factor pass the test with the precisely measured form factor for $D \rightarrow K e^{+} v_{e}$ decay, the uncertainty of the semileptonic $B$ decay form factor calculated in LQCD would be reduced. This would help in reducing the uncertainty of the measured $\left|V_{u b}\right|$ from semileptonic $B$ decays [1]. The improved measurement of $\left|V_{u b}\right|$ from semileptonic $B$ decay will improve the determination of the $B_{d}$ unitarity triangle, with which one can more precisely test the SM and search for NP.

In the past decades, copious measurements of branching fractions and/or decay rates for $D \rightarrow K e^{+} v_{e}$ decays were performed at more than ten experiments. By comprehensive analysis of these existing measurements together with $\left|V_{c s}\right|$ from SM global fit or together with form factor $f_{+}^{K}(0)$ calculated in LQCD, one can precisely determine the form factor $f_{+}^{K}(0)$ or extract $\left|V_{c s}\right|$.

In this article, we report the determination of $f_{+}^{K}(0)$ or extraction of $\left|V_{c s}\right|$ by analyzing all of these existing measurements of the semileptonic $D \rightarrow K e^{+} v_{e}$ decays in conjunction with $\left|V_{c s}\right|$ from SM global fit or with the form factor $f_{+}^{K}(0)$ calculated in LQCD. 
In the following sections, we first review the experimental measurements of branching fractions and decay rates for $D \rightarrow K e^{+} v_{e}$ decays in Sect. 2 . We then describe our comprehensive analysis procedure for dealing with these measurements to obtain the product of $f_{+}^{K}(0)$ and $\left|V_{c s}\right|$ in Sect. 3. In Sect. 4, we present the final results of our comprehensive analysis of these measurements. We finally give a summary for the determination of $f_{+}^{K}(0)$ and the extraction of $\left|V_{c S}\right|$ in Sect. 5.

\section{Experiments}

\subsection{Relative measurements}

In 1989, the Tagged Photon Spectrometer Collaboration studied the $D^{0} \rightarrow K^{-} e^{+} v_{e}$ decays and found $250 \mathrm{sig}$ nal events for $D^{0} \rightarrow K^{-} e^{+} v_{e}$ decays at the E691 experiment. Based on these events, they measured the ratio of decay rates $R_{0} \equiv \Gamma\left(D^{0} \rightarrow K^{-} e^{+} v_{e}\right) / \Gamma\left(D^{0} \rightarrow K^{-} \pi^{+}\right)=$ $0.91 \pm 0.07 \pm 0.11[2]$.

In 1991 , by analyzing $490 \mathrm{pb}^{-1}$ data collected with the CLEO detector at the Cornell Electron Storage Ring (CESR), the CLEO Collaboration made a measurement of the branching ratio of $D^{0}$ semileptonic decays. They observed $584 \pm 37 \pm 39$ signal events from $D^{0} \rightarrow K^{-} e^{+} v_{e}$ decays and obtained the ratio of branching fractions $R_{0} \equiv B\left(D^{0} \rightarrow\right.$ $\left.K^{-} e^{+} v_{e}\right) / B\left(D^{0} \rightarrow K^{-} \pi^{+}\right)=0.90 \pm 0.06 \pm 0.06$ [3].

In 1993, the CLEO Collaboration measured the branching ratios of the semileptonic $D$ decay modes using $1.68 \mathrm{fb}^{-1}$ data collected with the CLEO-II detector at the CESR. They selected the semileptonic $D$ decays from $e^{+} e^{-} \rightarrow c \bar{c}$ events and measured the ratios $R_{0} \equiv B\left(D^{0} \rightarrow K^{-} e^{+} v_{e}\right) / B\left(D^{0} \rightarrow\right.$ $\left.K^{-} \pi^{+}\right)=0.978 \pm 0.027 \pm 0.044$ and $R_{+} \equiv B\left(D^{+} \rightarrow\right.$ $\left.\bar{K}^{0} e^{+} v_{e}\right) / B\left(D^{+} \rightarrow \bar{K}^{0} \pi^{+}\right)=2.60 \pm 0.35 \pm 0.26$ [4].

In 2007, the BaBar Collaboration studied the $D^{0} \rightarrow$ $K^{-} e^{+} v_{e}$ decays by analyzing $75 \mathrm{fb}^{-1}$ data collected at $10.6 \mathrm{GeV}$ [5]. They selected $D^{0} \rightarrow K^{-} e^{+} v_{e}$ decays from $e^{+} e^{-} \rightarrow c \bar{c}$ events and divide the candidate events into ten $q^{2}$ bins. In each $q^{2}$ bin, the partial decay rate is measured relative to the normalization mode, $D^{0} \rightarrow K^{-} \pi^{+}$.

All above mentioned measurements are relative measurements which could not be used directly to determine the form factor $f_{+}^{K}(0)$ or $\left|V_{c s}\right|$. To use these measurements to determine $f_{+}^{K}(0)$ or $\left|V_{c S}\right|$, we should first transfer these measurements into absolute decay rates in certain $q^{2}$ range. The absolute decay rate $\Delta \Gamma$ can be obtained from the measured relative decay branching ratio $R$ by

$\Delta \Gamma=R \times B(D \rightarrow K \pi) \times \frac{1}{\tau_{D}}$,

where $B(D \rightarrow K \pi)$ is the branching fraction for $D^{0} \rightarrow$ $K^{-} \pi^{+}$or $D^{+} \rightarrow \bar{K}^{0} \pi^{+}$decays, and $\tau_{D}$ is the lifetime of $D$ meson.
Table 1 The partial rates $\Delta \Gamma$ of the $D^{0} \rightarrow K^{-} e^{+} v_{e}$ decays in $q^{2}$ ranges obtained from different experiments. $q_{\max }^{2}$ is the maximum value of $q^{2}$

\begin{tabular}{|c|c|c|}
\hline Experiment & $q^{2}\left(\mathrm{GeV} / c^{2}\right)$ & $\Delta \Gamma\left(\mathrm{ns}^{-1}\right)$ \\
\hline E691 [2] & $\left(0.0, q_{\max }^{2}\right)$ & $86.76 \pm 12.48$ \\
\hline CLEO [3] & $\left(0.0, q_{\max }^{2}\right)$ & $85.81 \pm 8.17$ \\
\hline CLEO-II [4] & $\left(0.0, q_{\max }^{2}\right)$ & $93.25 \pm 5.08$ \\
\hline \multirow[t]{10}{*}{ BaBar [5] } & $(0.0,0.2)$ & $17.75 \pm 0.48$ \\
\hline & $(0.2,0.4)$ & $16.26 \pm 0.49$ \\
\hline & $(0.4,0.6)$ & $14.42 \pm 0.42$ \\
\hline & $(0.6,0.8)$ & $12.39 \pm 0.38$ \\
\hline & $(0.8,1.0)$ & $9.92 \pm 0.31$ \\
\hline & $(1.0,1.2)$ & $7.72 \pm 0.26$ \\
\hline & $(1.2,1.4)$ & $5.32 \pm 0.21$ \\
\hline & $(1.4,1.6)$ & $3.24 \pm 0.14$ \\
\hline & $(1.6,1.8)$ & $1.29 \pm 0.09$ \\
\hline & $\left(1.8, q_{\max }^{2}\right)$ & $0.06 \pm 0.01$ \\
\hline Mark-III [15] & $\left(0.0, q_{\max }^{2}\right)$ & $82.91 \pm 15.62$ \\
\hline BES-II [16] & $\left(0.0, q_{\max }^{2}\right)$ & $93.15 \pm 11.77$ \\
\hline BES-III [18-20] & $\left(0.0, q_{\max }^{2}\right)$ & $85.47 \pm 0.93$ \\
\hline \multirow[t]{9}{*}{ CLEO-c [21] } & $(0.0,0.2)$ & $17.82 \pm 0.43$ \\
\hline & $(0.2,0.4)$ & $15.83 \pm 0.39$ \\
\hline & $(0.4,0.6)$ & $13.91 \pm 0.36$ \\
\hline & $(0.6,0.8)$ & $11.69 \pm 0.32$ \\
\hline & $(0.8,1.0)$ & $9.36 \pm 0.28$ \\
\hline & $(1.0,1.2)$ & $7.08 \pm 0.24$ \\
\hline & $(1.2,1.4)$ & $5.34 \pm 0.21$ \\
\hline & $(1.4,1.6)$ & $3.09 \pm 0.16$ \\
\hline & $\left(1.6, q_{\max }^{2}\right)$ & $1.28 \pm 0.11$ \\
\hline
\end{tabular}

To avoid the possible correlations, here we use the value of the branching fraction of $D^{0} \rightarrow K^{-} \pi^{+}$decay, $B\left(D^{0} \rightarrow\right.$ $\left.K^{-} \pi^{+}\right)=(3.91 \pm 0.05) \%$, which is the average of the measurements from BaBar [6], CLEO-c [7], CLEO-II [8], ALEPH $[9,10]$, and ARGUS [11]. For the branching fraction of $D^{+} \rightarrow \bar{K}^{0} \pi^{+}$decay, we use the value of $B\left(D^{+} \rightarrow\right.$ $\left.\bar{K}^{0} \pi^{+}\right)=(2.986 \pm 0.069) \%$, which is the sum of CLEOc's measurements $B\left(D^{+} \rightarrow K_{S}^{0} \pi^{+}\right)=(1.526 \pm 0.022 \pm$ $0.038) \%$ [7] and $B\left(D^{+} \rightarrow K_{L}^{0} \pi^{+}\right)=(1.460 \pm 0.040 \pm$ $0.035) \%[12]$.

Using the lifetime of $D$ meson, $\tau_{D^{0}}=(410.1 \pm 1.5) \times$ $10^{-15} \mathrm{~s}$, and $\tau_{D^{+}}=(1040 \pm 7) \times 10^{-15} \mathrm{~s}$ from PDG [13], the branching fractions of $B\left(D^{0} \rightarrow K^{-} \pi^{+}\right)=(3.91 \pm$ $0.05) \%$ and $B\left(D^{+} \rightarrow \bar{K}^{0} \pi^{+}\right)=(2.986 \pm 0.069) \%$, we translate these measurements of relative branching fractions and relative partial decay rates into absolute partial decay rates as shown in Tables 1 and 2.

In addition to the measurements of relative branching fractions and relative partial rates, the FOCUS Collaboration measured the non-parametric relative form factors 
Table 2 The partial rates of the $D^{+} \rightarrow \bar{K}^{0} e^{+} v_{e}$ decays in $q^{2}$ ranges obtained from different experiments. $q_{\max }^{2}$ is the maximum value of $q^{2}$

\begin{tabular}{lll}
\hline Experiment & $q^{2}\left(\mathrm{GeV} / c^{2}\right)$ & $\Delta \Gamma\left(\mathrm{ns}^{-1}\right)$ \\
\hline CLEO-II [4] & $\left(0.0, q_{\max }^{2}\right)$ & $74.65 \pm 12.65$ \\
BES-II [17] & $\left(0.0, q_{\max }^{2}\right)$ & $86.06 \pm 16.60$ \\
CLEO-c [21] & $(0.0,0.2)$ & $17.79 \pm 0.65$ \\
& $(0.2,0.4)$ & $15.62 \pm 0.59$ \\
& $(0.4,0.6)$ & $14.02 \pm 0.54$ \\
& $(0.6,0.8)$ & $12.28 \pm 0.49$ \\
& $(0.8,1.0)$ & $8.92 \pm 0.41$ \\
& $(1.0,1.2)$ & $8.17 \pm 0.37$ \\
& $(1.2,1.4)$ & $4.96 \pm 0.27$ \\
& $(1.4,1.6)$ & $2.67 \pm 0.19$ \\
& $\left(1.6, q_{\max }^{2}\right)$ & $1.19 \pm 0.13$ \\
\hline
\end{tabular}

Table 3 Measurements of normalized form factors $f_{+}^{K}\left(q_{i}^{2}\right) / f_{+}^{K}(0)$ at the FOCUS experiment

\begin{tabular}{lll}
\hline$i$ & $q_{i}^{2}\left(\mathrm{GeV} / c^{2}\right)$ & $f_{+}^{K}\left(q_{i}^{2}\right) / f_{+}^{K}(0)$ \\
\hline 1 & 0.09 & $1.01 \pm 0.03$ \\
2 & 0.27 & $1.11 \pm 0.05$ \\
3 & 0.45 & $1.15 \pm 0.07$ \\
4 & 0.63 & $1.17 \pm 0.08$ \\
5 & 0.81 & $1.24 \pm 0.09$ \\
6 & 0.99 & $1.45 \pm 0.09$ \\
7 & 1.17 & $1.47 \pm 0.11$ \\
8 & 1.35 & $1.48 \pm 0.16$ \\
9 & 1.53 & $1.84 \pm 0.19$ \\
\hline
\end{tabular}

$f_{+}^{K}\left(q^{2}\right) / f_{+}^{K}(0)$ at the central values of nine $q^{2}$ bins by analyzing the $D^{0} \rightarrow K^{-} \mu^{+} v_{\mu}$ decays in 2005 [14]. These measured variations of $f_{+}^{K}\left(q^{2}\right) / f_{+}^{K}(0)$ at FOCUS experiment also provide useful information about the semileptonic decay form factor and are helpful to determine the product $f_{+}^{K}(0)\left|V_{c s}\right|$ and the shape parameters of the form factor. These measurements are listed in Table 3 and are used in the further analysis.

\subsection{Absolute measurements}

In 1989, the Mark III Collaboration performed a measurement of absolute branching fraction for semileptonic $D^{0} \rightarrow K^{-} e^{+} v_{e}$ decay by analyzing data taken at the peak of $\psi(3770)$ resonance with the Mark III detector. They tagged $3636 \pm 54 \pm 195 \bar{D}^{0}$ mesons and found $55 D^{0} \rightarrow K^{-} e^{+} v_{e}$ signal events in the system recoiling against the $\bar{D}^{0}$ tags. With these events, they measured the absolute decay branch- ing fraction $B\left(D^{0} \rightarrow K^{-} e^{+} v_{e}\right)=(3.4 \pm 0.5 \pm 0.4)$ $\%$ [15].

Using the similar method as the one used in Mark III, the BES-II Collaboration measured the branching fractions of $D \rightarrow K e^{+} v_{e}$ decays by analyzing about $33 \mathrm{pb}^{-1}$ data taken near $3.773 \mathrm{GeV}$ with the BES-II detector at the BEPC collider. Their results are $B\left(D^{0} \rightarrow K^{-} e^{+} v_{e}\right)=(3.82 \pm$ $0.40 \pm 0.27) \%[16]$ and $B\left(D^{+} \rightarrow \bar{K}^{0} e^{+} v_{e}\right)=(8.95 \pm$ $1.59 \pm 0.67) \%[17]$.

Recently, the BESIII Collaboration reported preliminary results of $D^{0} \rightarrow K^{-} e^{+} v_{e}$ decays obtained by analyzing $2.92 \mathrm{fb}^{-1}$ data taken at $3.773 \mathrm{GeV}$. They accumulated $(279.3 \pm 0.4) \times 10^{4} \bar{D}^{0}$ tags from five hadronic decay modes. In this sample of $\bar{D}^{0}$ tags, they observed $70727 \pm 278$ signal events for $D^{0} \rightarrow K^{-} e^{+} v_{e}$ decays and measured the branching fraction $B\left(D^{0} \rightarrow K^{-} e^{+} v_{e}\right)=(3.505 \pm 0.014 \pm$ $0.033) \%[18-20]$.

The partial decay rate is related to the decay branching fraction by

$\Delta \Gamma=B\left(D \rightarrow K e^{+} v_{e}\right) \times \frac{1}{\tau_{D}}$.

Using the lifetimes of $D^{0}$ and $D^{+}$mesons quoted from PDG [13], $\tau_{D^{0}}=(410.1 \pm 1.5) \times 10^{-15} \mathrm{~s}$ and $\tau_{D^{+}}=(1040 \pm$ 7) $\times 10^{-15} \mathrm{~s}$, we translate these absolute measurements of branching fractions for $D \rightarrow K e^{+} v_{e}$ decays into the partial decay rates, which are shown in Tables 1 and 2.

In 2009, the CLEO Collaboration studied the semileptonic decays of $D^{0} \rightarrow K^{-} e^{+} v_{e}$ and $D^{+} \rightarrow \bar{K}^{0} e^{+} v_{e}$ by analyzing $818 \mathrm{pb}^{-1}$ data collected at $3.773 \mathrm{GeV}$ with the CLEO-c detector. Using double tag method, they measured the decay rates for semileptonic $D^{0} \rightarrow K^{-} e^{+} v_{e}$ and $D^{+} \rightarrow \bar{K}^{0} e^{+} v_{e}$ decays in nine $q^{2}$ bins [21]. These measurements of decay rates are summarized in Tables 1 and 2 .

In 2006, the Belle Collaboration published the results on the $D^{0} \rightarrow K^{-} \ell^{+} v_{\ell}$ decays. They accumulated $56461 \pm$ $309 \pm 830$ inclusive $D^{0}$ mesons and found $1318 \pm 37 \pm 7$ signal events for $D^{0} \rightarrow K^{-} e^{+} v_{e}$ decays and $1249 \pm 37 \pm 25$ signal events for $D^{0} \rightarrow K^{-} \mu^{+} v_{\mu}$ decays from a $282 \mathrm{fb}^{-1}$ data set collected around $10.58 \mathrm{GeV}$ with the Belle detector [22]. Using these selected events from semileptonic $D^{0}$ decays, they obtained the form factors $f_{+}^{K}\left(q^{2}\right)$ in $27 q^{2}$ bins with the bin size of $0.067 \mathrm{GeV}^{2} / c^{4}$. To obtain the product $f_{+}^{K}\left(q_{i}^{2}\right)\left|V_{c s}\right|$ which will be used in our comprehensive analysis in Sect. 3, we extrapolate these measurements of form factors at the Belle experiment to the product $f_{+}^{K}\left(q_{i}^{2}\right)\left|V_{c s}\right|$ using the PDG'2006 value of $\left|V_{c s}\right|=0.97296 \pm 0.00024$ [23] which was originally used in the Belle's paper published. Table 4 lists the form factors $f_{+}^{K}\left(q_{i}^{2}\right)$ measured at the Belle experiment and our translated products $f_{+}^{K}\left(q_{i}^{2}\right)\left|V_{c s}\right|$. These products will be used in our further analysis described in Sect. 3. 
Table 4 Measurements of form factors $f_{+}^{K}\left(q_{i}^{2}\right)$ at the Belle experiment and the products $f_{+}^{K}\left(q_{i}^{2}\right)\left|V_{c s}\right|$

\begin{tabular}{llll}
\hline$i$ & $q_{i}^{2}\left(\mathrm{GeV} / c^{2}\right)$ & $f_{+}^{K}\left(q_{i}^{2}\right)$ & $f_{+}^{K}\left(q_{i}^{2}\right)\left|V_{c s}\right|$ \\
\hline 1 & 0.100 & $0.707 \pm 0.030$ & $0.688 \pm 0.029$ \\
2 & 0.167 & $0.783 \pm 0.030$ & $0.762 \pm 0.029$ \\
3 & 0.233 & $0.763 \pm 0.030$ & $0.743 \pm 0.029$ \\
4 & 0.300 & $0.833 \pm 0.033$ & $0.811 \pm 0.032$ \\
5 & 0.367 & $0.783 \pm 0.033$ & $0.762 \pm 0.032$ \\
6 & 0.433 & $0.840 \pm 0.037$ & $0.817 \pm 0.036$ \\
7 & 0.500 & $0.880 \pm 0.040$ & $0.856 \pm 0.039$ \\
8 & 0.567 & $0.940 \pm 0.040$ & $0.915 \pm 0.039$ \\
9 & 0.633 & $0.907 \pm 0.040$ & $0.882 \pm 0.039$ \\
10 & 0.700 & $0.820 \pm 0.040$ & $0.798 \pm 0.039$ \\
11 & 0.767 & $1.023 \pm 0.043$ & $0.996 \pm 0.042$ \\
12 & 0.833 & $0.997 \pm 0.047$ & $0.970 \pm 0.045$ \\
13 & 0.900 & $0.947 \pm 0.047$ & $0.921 \pm 0.045$ \\
14 & 0.967 & $1.043 \pm 0.053$ & $1.015 \pm 0.052$ \\
15 & 1.033 & $1.100 \pm 0.053$ & $1.070 \pm 0.052$ \\
16 & 1.100 & $0.937 \pm 0.057$ & $0.911 \pm 0.055$ \\
17 & 1.167 & $1.113 \pm 0.067$ & $1.083 \pm 0.065$ \\
18 & 1.233 & $1.097 \pm 0.070$ & $1.067 \pm 0.068$ \\
19 & 1.300 & $1.253 \pm 0.080$ & $1.219 \pm 0.078$ \\
20 & 1.367 & $1.380 \pm 0.087$ & $1.343 \pm 0.084$ \\
21 & 1.433 & $1.313 \pm 0.103$ & $1.278 \pm 0.101$ \\
22 & 1.500 & $1.190 \pm 0.110$ & $1.158 \pm 0.107$ \\
23 & 1.567 & $1.417 \pm 0.123$ & $1.378 \pm 0.120$ \\
24 & 1.633 & $1.473 \pm 0.173$ & $1.433 \pm 0.169$ \\
25 & 1.700 & $1.413 \pm 0.220$ & $1.375 \pm 0.214$ \\
26 & 1.767 & $1.147 \pm 0.340$ & $1.116 \pm 0.331$ \\
27 & 1.833 & & $1.411 \pm 0.892$ \\
\hline & &
\end{tabular}

\section{Analysis}

To obtain the product of the hadronic form factor at four momentum transfer $q=0, f_{+}^{K}(0)$, and the magnitude of CKM matrix element $\left|V_{c s}\right|$, we perform a comprehensive $\chi^{2}$ fit to these experimental measurements of the partial decay rates. The object function to be minimized in the fit is defined as

$\chi^{2}=\chi_{\mathrm{R}}^{2}+\chi_{\mathrm{P}}^{2}+\chi_{\mathrm{F}}^{2}$,

where $\chi_{\mathrm{R}}^{2}$ is for these measurements of decay branching fraction and/or partial decay rates in different $q^{2}$ ranges, $\chi_{\mathrm{P}}^{2}$ corresponds to the products of $f_{+}^{K}\left(q_{i}^{2}\right)\left|V_{c s}\right|$ measured at the Belle experiment, and $\chi_{\mathrm{F}}^{2}$ is built for the measurements of $f_{+}^{K}\left(q_{i}^{2}\right) / f_{+}^{K}(0)$ measured at the FOCUS experiment.

Taking into account the correlations between these measurements, the quantity $\chi_{\mathrm{R}}^{2}$ is given by
$\chi_{\mathrm{R}}^{2}=\sum_{i=1}^{36} \sum_{j=1}^{36}\left(\Delta \Gamma_{i}^{\mathrm{ex}}-\Delta \Gamma_{i}^{\mathrm{th}}\right)\left(\mathcal{C}_{\mathrm{R}}^{-1}\right)_{i j}\left(\Delta \Gamma_{j}^{\mathrm{ex}}-\Delta \Gamma_{j}^{\mathrm{th}}\right)$,

where $\Delta \Gamma^{\mathrm{ex}}$ denotes the experimentally measured partial decay rate, $\Delta \Gamma^{\text {th }}$ is the theoretical expectation of the decay rate, and $\mathcal{C}_{\mathrm{R}}^{-1}$ is the inverse of the covariance matrix $\mathcal{C}_{\mathrm{R}}$, which is a $36 \times 36$ matrix containing the correlations between the measured partial decay rates. The construction of $C_{\mathrm{R}}$ is discussed in Sect. 3.2.

With the parametrization of the form factor, the theoretically predicted partial decay rate in a given $q^{2}$ bin is obtained by integrating Eq. (1) from the low boundary $q_{\text {low }}^{2}$ to the up boundary $q_{\mathrm{up}}^{2}$ of the $q^{2}$ bin,

$\Delta \Gamma^{\text {th }}=\int_{q_{\text {low }}^{2}}^{q_{\text {up }}^{2}} \frac{G_{F}^{2}}{24 \pi^{3}}\left|V_{c S}\right|^{2} p^{3}\left|f_{+}^{K}\left(q^{2}\right)\right|^{2} d q^{2}$.

In this analysis, we used several forms of the form factor parameterizations which are discussed in Sect. 3.1.

Ignoring some possible correlations of the measurements of the product $f_{+}^{K}\left(q_{i}^{2}\right)\left|V_{c s}\right|$ measured at the Belle experiment, the function $\chi_{\mathrm{P}}^{2}$ in Eq. (4) is defined as

$\chi_{\mathrm{P}}^{2}=\sum_{i=1}^{27}\left(\frac{\tilde{f}_{i}^{\mathrm{ex}}-\tilde{f}_{i}^{\mathrm{th}}}{\sigma_{i}}\right)^{2}$,

where $\tilde{f}_{i}^{\text {ex }}$ is the measured product $f_{+}^{K}\left(q^{2}\right)\left|V_{c S}\right|$ at the center of $i$ th $q^{2}$ bin $q_{i}^{2}$ with the standard deviation $\sigma_{i}$, and $\tilde{f}_{i}^{\text {th }}$ is the theoretical expectation of the product $f_{+}^{K}\left(q^{2}\right)\left|V_{c s}\right|$ at $q_{i}^{2}$.

Considering the correlations of the non-parametric form factors measured at the FOCUS experiment, the $\chi_{\mathrm{F}}^{2}$ is constructed as

$\chi_{\mathrm{F}}^{2}=\sum_{i=1}^{9} \sum_{j=1}^{9}\left(F_{i}^{\mathrm{ex}}-F_{i}^{\mathrm{th}}\right)\left(\mathcal{C}_{\mathrm{F}}^{-1}\right)_{i j}\left(F_{j}^{\mathrm{ex}}-F_{j}^{\mathrm{th}}\right)$,

where $F_{i}^{\text {ex }}$ is the measured relative form factor $f_{+}^{K}\left(q_{i}^{2}\right) / f_{+}^{K}(0)$ at $q_{i}^{2}$ from the FOCUS experiment, $F_{i}^{\text {th }}$ is the theoretically expected value of $f_{+}^{K}\left(q_{i}^{2}\right) / f_{+}^{K}(0)$, and $\mathcal{C}_{\mathrm{F}}^{-1}$ is the inverse of the covariance matrix $\mathcal{C}_{\mathrm{F}}$. The construction of $\mathcal{C}_{\mathrm{F}}$ is described later in the Sect. 3.2.

\subsection{Form factor parameterizations}

In general, the single pole model is the simplest approach to describe the $q^{2}$ dependent behavior of form factor. The single pole model is expressed as

$f_{+}^{K}\left(q^{2}\right)=\frac{f_{+}^{K}(0)}{1-q^{2} / m_{\text {pole }}^{2}}$, 
where $f_{+}^{K}(0)$ is the value of form factor at $q^{2}=0, m_{\text {pole }}$ is the pole mass which is predicted to be the mass of the $D_{s}^{*+}$ meson for semileptonic $D \rightarrow K e^{+} v_{e}$ decays.

The so-called BK parameterization [24] is also widely used in LQCD calculations and experimental studies of this decay. In the BK parameterization, the form factor of the semileptonic $D \rightarrow K e^{+} v_{e}$ decays is written as

$f_{+}^{K}\left(q^{2}\right)=\frac{f_{+}^{K}(0)}{\left(1-q^{2} / m_{D_{s}^{*+}}^{2}\right)\left(1-\alpha q^{2} / m_{D_{s}^{*+}}^{2}\right)}$,

where $m_{D_{s}^{*+}}$ is the mass of the $D_{s}^{*+}$ meson, and $\alpha$ is a free parameter to be fitted. The value of $\alpha$ is assumed to be around 1.75 for $D \rightarrow K \ell^{+} \nu_{\ell}$ in the BK parameterization.

The ISGW2 model [25] assumes

$f_{+}^{K}\left(q^{2}\right)=f_{+}^{K}\left(q_{\max }^{2}\right)\left(1+\frac{r^{2}}{12}\left(q_{\max }^{2}-q^{2}\right)\right)^{-2}$,

where $q_{\max }^{2}$ is the kinematical limit of $q^{2}$, and $r$ is the conventional radius of the meson. In this model, the predictions of $f_{+}^{K}\left(q_{\max }^{2}\right)$ and $r$ for $D \rightarrow K \ell^{+} \nu_{\ell}$ decays are 1.23 and $1.12 \mathrm{GeV}^{-1} c^{2}$, respectively.

The most general parameterization of the form factor is the series expansion [26], which is based on analyticity and unitarity. In this parametrization, the variable $q^{2}$ is mapped to a new variable $z$ through

$z\left(q^{2}, t_{0}\right)=\frac{\sqrt{t_{+}-q^{2}}-\sqrt{t_{+}-t_{0}}}{\sqrt{t_{+}-q^{2}}+\sqrt{t_{+}-t_{0}}}$

with $t_{ \pm}=\left(m_{D} \pm m_{K}\right)^{2}$ and $t_{0}=t_{+}\left(1-\sqrt{1-t_{-} / t_{+}}\right)$. The form factor is then expressed in terms of the new variable $z$ as

$f_{+}^{K}\left(q^{2}\right)=\frac{1}{P\left(q^{2}\right) \phi\left(q^{2}, t_{0}\right)} \sum_{k=0}^{\infty} a_{k}\left(t_{0}\right)\left[z\left(q^{2}, t_{0}\right)\right]^{k}$,

where $P\left(q^{2}\right)=z\left(q^{2}, m_{D_{s}^{*+}}^{2}\right)$ which accounts for the presence of the pole, $\phi\left(q^{2}, t_{0}\right)$ is an arbitrary function, and $a_{k}\left(t_{0}\right)$ are real coefficients. In this analysis, the choice of $\phi\left(q^{2}, t_{0}\right)$ is taken to be

$$
\begin{aligned}
\phi\left(q^{2}, t_{0}\right)= & \left(\frac{\pi m_{c}^{2}}{3}\right)^{\frac{1}{2}}\left(\frac{z\left(q^{2}, 0\right)}{-q^{2}}\right)^{\frac{5}{2}}\left(\frac{z\left(q^{2}, t_{0}\right)}{t_{0}-q^{2}}\right)^{-\frac{1}{2}} \\
& \times\left(\frac{z\left(q^{2}, t_{-}\right)}{t_{-}-q^{2}}\right)^{-\frac{3}{4}} \frac{\left(t_{+}-q^{2}\right)}{\left(t_{+}-t_{0}\right)^{\frac{1}{4}}},
\end{aligned}
$$

where $m_{c}$ is the mass of charm quark, which is taken to be $1.2 \mathrm{GeV} / c^{2}$.
In practical use, one usually make a truncation on the above series. Actually, it is found that the current experimental data can be adequately described by only the first three terms in Eq. (13).

In this analysis we will fit the measured decay rates to the three-parameter series expansion. After optimizing the form factor parameters, we obtain the form for the three-parameter series expansion:

$f_{+}^{K}\left(q^{2}\right)=\frac{f_{+}^{K}(0) P(0) \phi\left(0, t_{0}\right)\left(1+\sum_{k=1}^{2} r_{k}\left[z\left(q^{2}, t_{0}\right)\right]^{k}\right)}{P\left(q^{2}\right) \phi\left(q^{2}, t_{0}\right)\left(1+\sum_{k=1}^{2} r_{k}\left[z\left(0, t_{0}\right)\right]^{k}\right)}$,

where $r_{k} \equiv a_{k}\left(t_{0}\right) / a_{0}\left(t_{0}\right)(k=1,2)$.

\subsection{Covariance matrix}

It's a little complicated to compute the covariances of these 36 partial decay rates measured in different $q^{2}$ ranges and at different experiments. To be clear, we separate the correlations among these $\Delta \Gamma$ measurements into two case: the one associated with the experimental status of each independent experiment, and the other related to the external inputs of parameters such as the lifetime of the $D$ meson.

The statistical uncertainties in the $\Delta \Gamma$ measurements from the same experiment are correlated to some extent, while these are independent for the measurements from different experiments. The systematic uncertainties from tracking, particle identification, etc. are usually independent between different experiments. In this analysis, we treat the systematic uncertainties except the ones from $D$ lifetimes and branching fractions as fully uncorrelated between the measurements performed at different experiments. We consider these below:

- The covariances of the $\Delta \Gamma$ measured at the same experiment are computed using the statistical errors, the systematic errors, and the correlation coefficients, which are presented in their original papers published.

- For the measurements of $D^{0} \rightarrow K^{-} e^{+} v_{e}$ decay, the lifetime of $D^{0}$ meson is used to obtain the partial decay rates in particular $q^{2}$ ranges. The systematic uncertainties due to imperfect knowledge of $D^{0}$ lifetime are fully correlated among all these measurements of the partial rates of $D^{0} \rightarrow K^{-} e^{+} v_{e}$ decay. Similarly, the systematic uncertainties related to $D^{+}$lifetime are fully correlated among all of the $\Delta \Gamma$ measurements for $D^{+} \rightarrow \bar{K}^{0} e^{+} v_{e}$ decay.

- An additional systematic uncertainty from $B\left(D^{0} \rightarrow\right.$ $K^{-} \pi^{+}$) is fully correlated between these relative measurements of $D^{0} \rightarrow K^{-} e^{+} v_{e}$ decay at the E691, CLEO, CLEO-II and BaBar experiments. Since we only use 
Table 5 Fitted parameters corresponding to different form factor parameterizations and $\chi^{2} /$ d.o.f. of the fit

\begin{tabular}{lllr}
\hline Parameterization & $f_{+}^{K}(0)\left|V_{c s}\right|$ & Shape parameters & $\chi^{2} /$ d.o.f. \\
\hline Single pole & $0.720 \pm 0.003$ & $m_{\text {pole }}=(1.909 \pm 0.011) \mathrm{GeV} / c^{2}$ & $106.0 / 70$ \\
BK & $0.716 \pm 0.003$ & $\alpha=0.327 \pm 0.021$ & $101.0 / 70$ \\
ISGW2 & $0.714 \pm 0.003$ & $r=(1.610 \pm 0.015) \mathrm{GeV}^{-1} c^{2}$ & $101.9 / 70$ \\
Series expansion & $0.717 \pm 0.004$ & $r_{1}=-2.34 \pm 0.17$ & $101.1 / 69$ \\
& & $r_{2}=0.43 \pm 3.82$ & \\
\hline
\end{tabular}

one relative measurement of $D^{+} \rightarrow \bar{K}^{0} e^{+} v_{e}$ decay which is from the CLEO-II experiment, there are no correlations due to the normalization branching fraction $B\left(D^{+} \rightarrow \bar{K}^{0} \pi^{+}\right)$between this measurement and other measurements.

With these considerations mentioned above, we then construct a $36 \times 36$ covariance matrix $\mathcal{C}_{\mathrm{R}}$ which is necessary in the form factor fit.

The entry in $i$ th row and $j$ th column of the covariance matrix $\mathcal{C}_{\mathrm{R}}$ in Eq. (8) is given by $\left(\mathcal{C}_{\mathrm{R}}\right)_{i j}=\sigma_{i} \sigma_{j} \rho_{i j}$, where $\sigma_{i}$ and $\sigma_{j}$ are the errors of the $f_{+}^{K}\left(q^{2}\right) / f_{+}^{K}(0)$ at $q_{i}^{2}$ and $q_{j}^{2}$ measured at the FOCUS experiment, respectively, and $\rho_{i j}$ is the correlation coefficient of these two measurements of $f_{+}^{K}\left(q^{2}\right) / f_{+}^{K}(0)$. The values of the errors and correlation coefficients are directly quoted from Ref. [14].

\subsection{Fits to experimental data}

Four fits are applied to the experimental data with the form factor hypothesis of single pole model, modified pole model, ISGW2 model and series expansion. The fit to experimental data returns the normalization $f_{+}^{K}(0)\left|V_{c s}\right|$ and the shape parameters of the form factor which govern the behavior of form factor in high $q^{2}$ range.

The numerical results of the fit corresponding to each form of the form factor parameterization are summarized in Table 5. As an example, Fig. 1 presents the result of the fit in the case of using the form factor parameterization of series expansion. In Fig. 1a and b, we compare the measured branching fractions of $D^{0} \rightarrow K^{-} e^{+} v_{e}$ and $D^{+} \rightarrow \bar{K}^{0} e^{+} v_{e}$ decays from different experiments. Figure $1 \mathrm{c}$ and d show the measured differential decay rates for $D^{0} \rightarrow K^{-} e^{+} \nu_{e}$ and $D^{+} \rightarrow \bar{K}^{0} e^{+} v_{e}$, respectively. Figure 1e depicts the measurements of $f_{+}^{K}\left(q^{2}\right)\left|V_{c s}\right|$ at different $q^{2}$ from the Belle experiments. The FOCUS measurements of the normalized form factor $f_{+}^{K}\left(q^{2}\right) / f_{+}^{K}(0)$ are illustrated in Fig. 1f. In these figures, the lines show the best fit to these measurements.

To check the fit quality and also the isospin invariance, the experimentally measured decay branching fractions and/or partial rates are mapped into the product $f_{+}^{K}\left(q_{i}^{2}\right)\left|V_{c s}\right|$ via
$f_{+}^{K}(0)\left|V_{c s}\right|=\sqrt{\frac{B}{\tau_{D}} \frac{1}{N}}$

and

$f_{+}^{K}\left(q_{i}^{2}\right)\left|V_{c s}\right|=\sqrt{\left(\frac{d \Gamma}{d q^{2}}\right)_{i} \frac{24 \pi^{3}}{G_{F}^{2} \boldsymbol{p}_{i}^{3}}}$,

where $B$ denotes the measured branching fraction, the differential decay rate $\left(d \Gamma / d q^{2}\right)_{i}$ is obtained by dividing measured decay rate in $q^{2}$ bin $i$ by the corresponding bin size. The normalization $N$ is given by

$N=\frac{G_{F}^{2}}{24 \pi^{3}\left|f_{+}^{K}(0)\right|^{2}} \int_{0}^{q_{\max }^{2}} p^{3}\left|f_{+}^{K}\left(q^{2}\right)\right|^{2} d q^{2}$.

The effective $p_{i}^{3}$ in $q^{2}$ bin $i$ is given by

$\boldsymbol{p}_{i}^{3}=\frac{\int_{q_{\mathrm{low}}^{2}}^{q_{\mathrm{u}}^{2}} \boldsymbol{p}^{3}\left|f_{+}^{K}\left(q^{2}\right)\right|^{2} d q^{2}}{\left|f_{+}^{K}\left(q_{i}^{2}\right)\right|^{2}\left(q_{\mathrm{up}}^{2}-q_{\mathrm{low}}^{2}\right)}$.

To calculate the integral in Eqs. (18) and (19), we use the shape parameters of form factor, which is obtained from the series expansion fit to the data.

Figure 2 shows the product $f_{+}^{K}\left(q^{2}\right)\left|V_{c s}\right|$ as a function of $q^{2}$, where the blue curve corresponds to the best series expansion fit to the experimental data. In this fit, eight measurements of $f_{+}^{K}(0)\left|V_{c s}\right|$ locate at $q^{2}=0$, which overlap each other. To be clear, these $f_{+}^{K}(0)\left|V_{c s}\right|$ translated from the decay branching fractions measured at different experiments are also displayed in the insert plot in Fig. 2.

\section{Results}

In this analysis, we choose the result from the fit using series expansion as our primary results and use this to extract the form factor $f_{+}^{K}(0)$ and the magnitude of the CKM matrix element $V_{c s}$. 

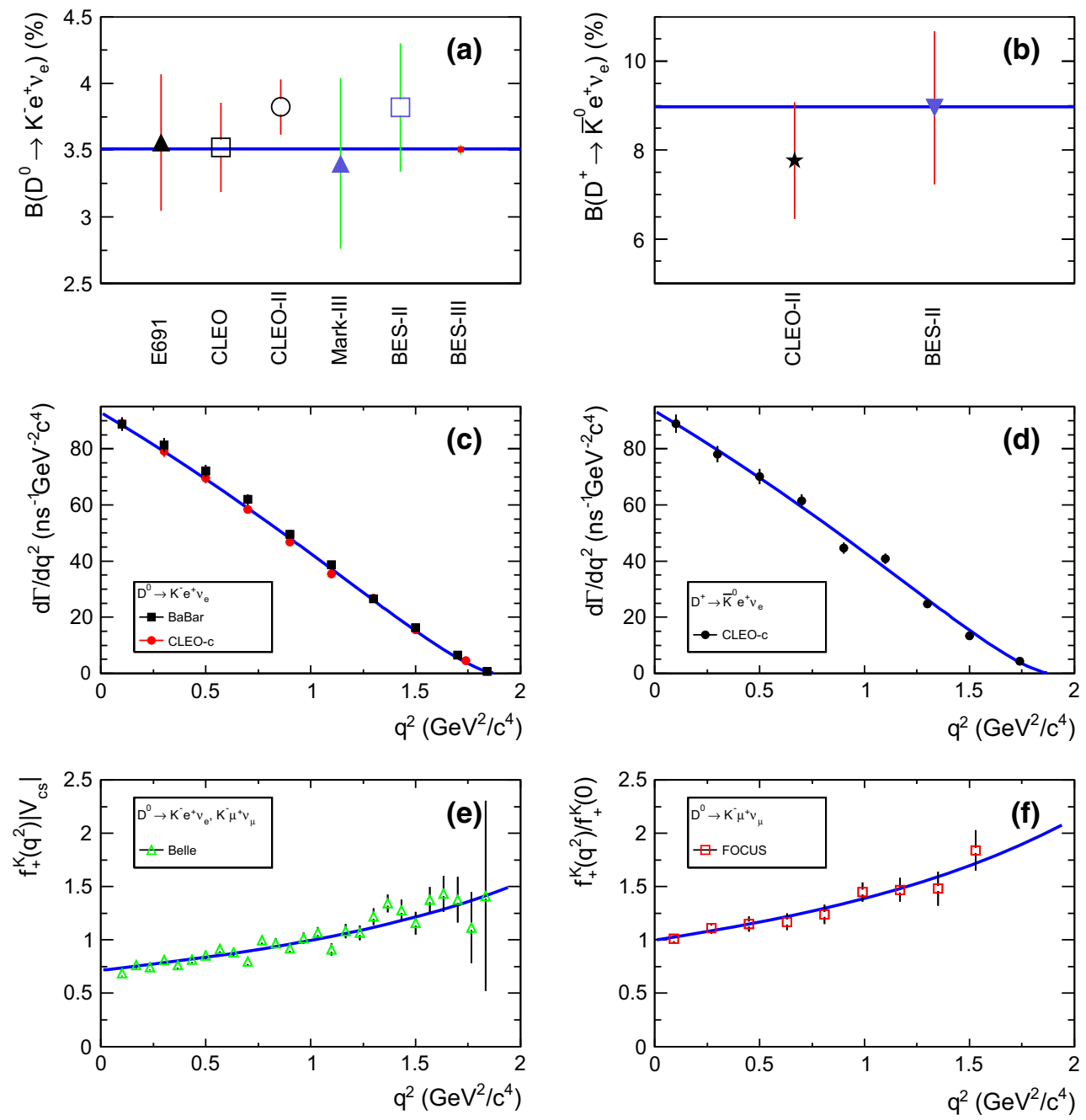

Fig. 1 Comparisons of branching fraction measurements for a $D^{0} \rightarrow$ $K^{-} e^{+} v_{e}, \mathbf{b} D^{+} \rightarrow \bar{K}^{0} e^{+} v_{e}, \mathbf{c}$ measurements of differential decay rates for $D^{0} \rightarrow K^{-} e^{+} v_{e}$ measured at the BaBar and CLEO-c experiments, d differential decay rates for $D^{+} \rightarrow \bar{K}^{0} e^{+} v_{e}$ measured at the CLEO-c

experiment, e the product of form factor and $\left|V_{c s}\right|$ measured at the Belle experiment, and $\mathbf{f}$ the normalized form factor measured at the FOCUS experiment. The blue lines show the fit to these measurements using the series expansion for the form factor

\subsection{Form factor $f_{+}^{K}(0)$}

Dividing the value of $f_{+}^{K}(0)\left|V_{c s}\right|=0.717 \pm 0.004$ shown in Table 5 from the series expansion fit by the $\left|V_{c s}\right|=0.97343 \pm$ 0.00015 obtained using unitarity constraints [13] yields the form factor

$f_{+}^{K}(0)=0.737 \pm 0.004 \pm 0.000$,

where the first uncertainty is from the combined statistical and systematic uncertainties in the partial decay rate measurements, and the second is due to the uncertainty in the $\left|V_{c s}\right|$.

The result for the form factor determined in this analysis is compared with the theoretical calculations of the form factor from the LQCD [27-29] and from QCD light-cone sum

rules [30] in Fig. 3. Our result of the form factor extracted by analyzing all existing experimental measurements is consistent with these values predicted by theory, but is with higher precision than the most accurate one from LQCD calculation by a factor of 2.8 .

\subsection{Parameters of form factor}

When these shape parameters of the form factor parameterization are left free in the fit, the form factor parametrizations of the single pole model, BK model, the ISGW2 model, and the series expansion model are all capable of describing the experimental data with almost identical $\chi^{2}$ probability. However, for the physical interpretation of the shape parameters in the single pole model, BK model, the ISGW2 model, the values of the parameters obtained from the fits are largely 


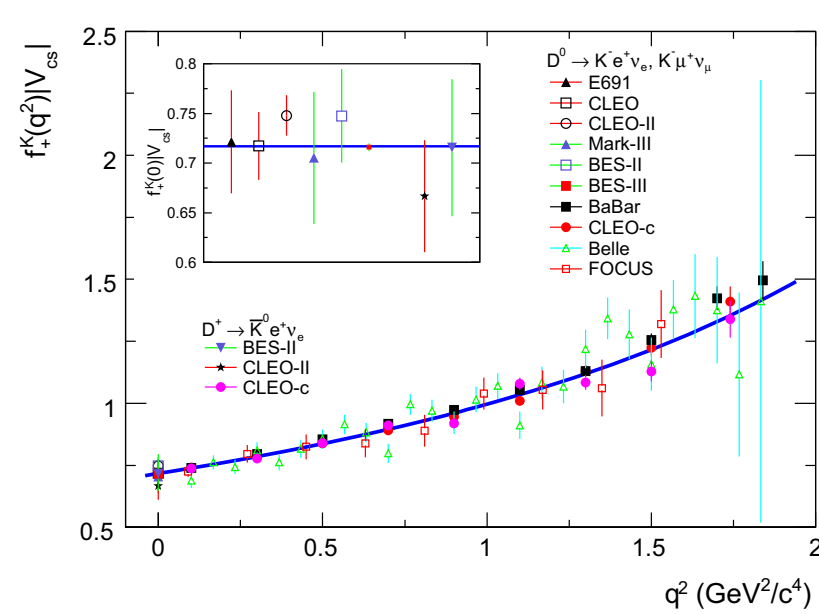

Fig. 2 The product $f_{+}^{K}\left(q^{2}\right)\left|V_{c s}\right|$ measured at different experiments as a function of $q^{2}$. The blue curve represents the series expansion fit to these $f_{+}^{K}\left(q^{2}\right)\left|V_{c s}\right|$. The insert plot shows the comparison of the products $f_{+}^{K}(0)\left|V_{c s}\right|$ which are obtained using the branching fractions measured at different experiments

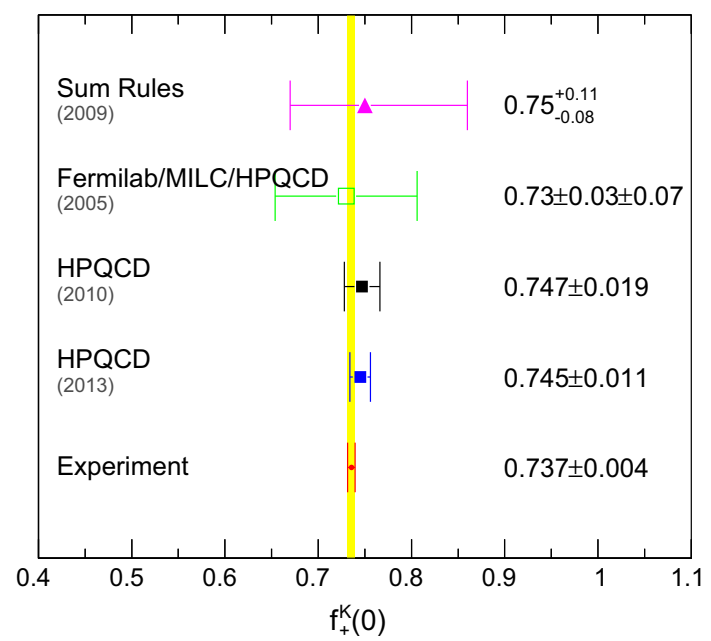

Fig. 3 Comparison of our determined form factor from experimental measurements with the theoretical calculations of the form factor

deviated from those expected values by these models. This indicates that the experimental data do not support the physical interpretation of the shape parameters in these parameterizations. Figure $4 \mathrm{a}-\mathrm{c}$ show the comparisons between the measured values and the theoretically expected values for the pole mass $m_{\text {pole }}$ in single pole model, $\alpha$ in BK model, and $r$ in ISGW2 model. These measured parameters do not agree with the values predicted by these form factor models.

\subsection{CKM matrix element $\left|V_{c s}\right|$}

Using the product $f_{+}^{K}(0)\left|V_{c s}\right|=0.717 \pm 0.004$ obtained from the comprehensive series expansion fit in conjunction with the form factor $f_{+}^{K}(0)=0.745 \pm 0.011$ [27] calculated in LQCD for the $D \rightarrow K$ transition, we determine the
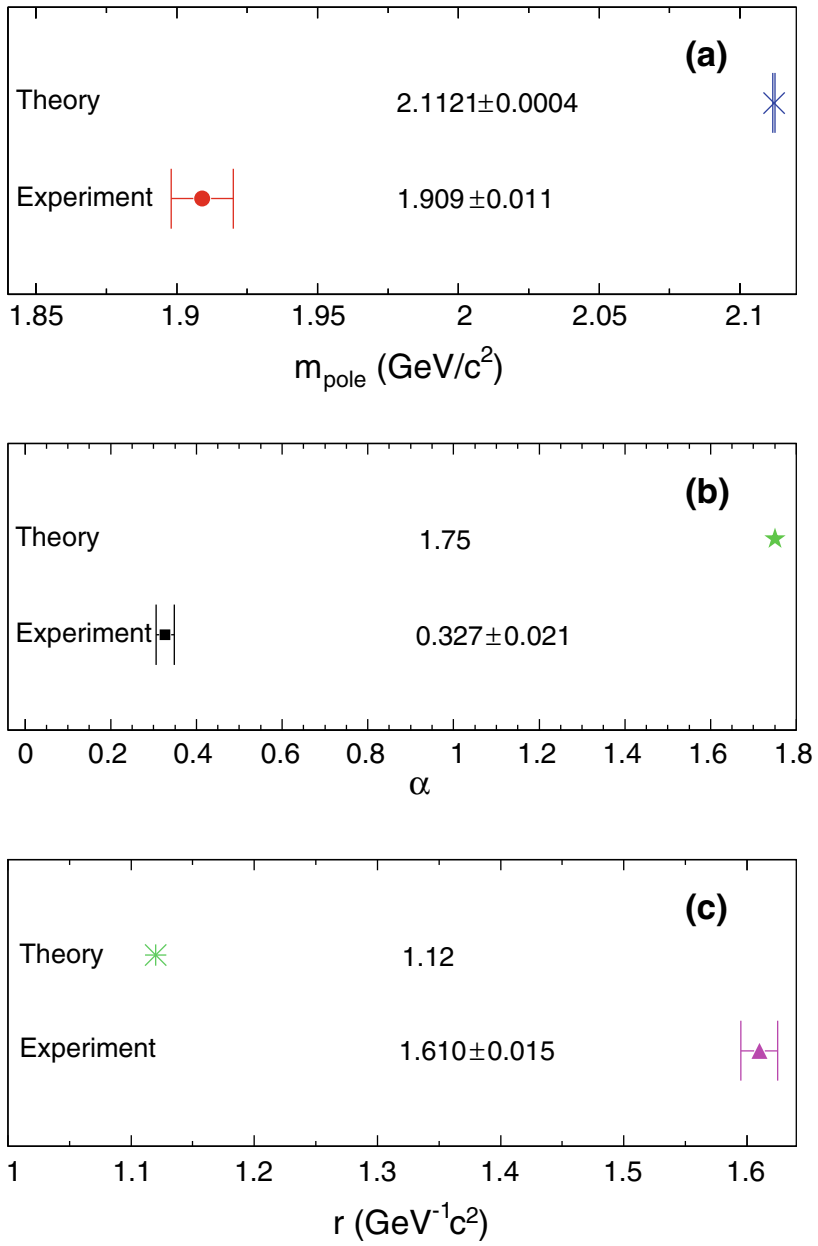

Fig. 4 Comparisons of the form factor parameters determined from experimental measurements and the theoretical expectations: a the pole mass $m_{\text {pole }}$ in single pole model, $\mathbf{b} \alpha$ in the BK model, and $\mathbf{c} r$ in the ISGW2 model

magnitude of the CKM matrix element $V_{c s}$ to be

$\left|V_{c s}\right|^{D \rightarrow K e^{+} v_{e}}=0.962 \pm 0.005 \pm 0.014$,

where the last uncertainty corresponds to the accuracy of the form factor $f_{+}^{K}(0)$ calculated in LQCD.

Combining with the value $\left|V_{c S}\right|_{s}^{D_{s}^{+} \rightarrow \ell^{+} v_{\ell}}=1.012 \pm$ $0.015 \pm 0.009$, which is extracted from the measurements of leptonic $D_{s}^{+}$decays (see Appendix A), we obtain the magnitude of the CKM matrix element $V_{c s}$ to be

$\left|V_{c s}\right|=0.983 \pm 0.011$

Figure 5 shows the comparisons of the value of $\left|V_{c s}\right|$ which is determined with the $\left|V_{c S}\right|^{D \rightarrow K e^{+} v_{e}}$ in this analysis together with the $\left|V_{c S}\right|_{s}^{+} \rightarrow \ell^{+} \nu_{\ell}$ determined from leptonic $D_{s}^{+}$decays, and the value from a SM global fit [13].

Figure 6 shows a comparison of our extracted $\left|V_{c s}\right|$ from all existing measurements of $D \rightarrow K e^{+} \nu_{e}$ and $D_{s}^{+} \rightarrow \ell^{+} v_{\ell}$ 


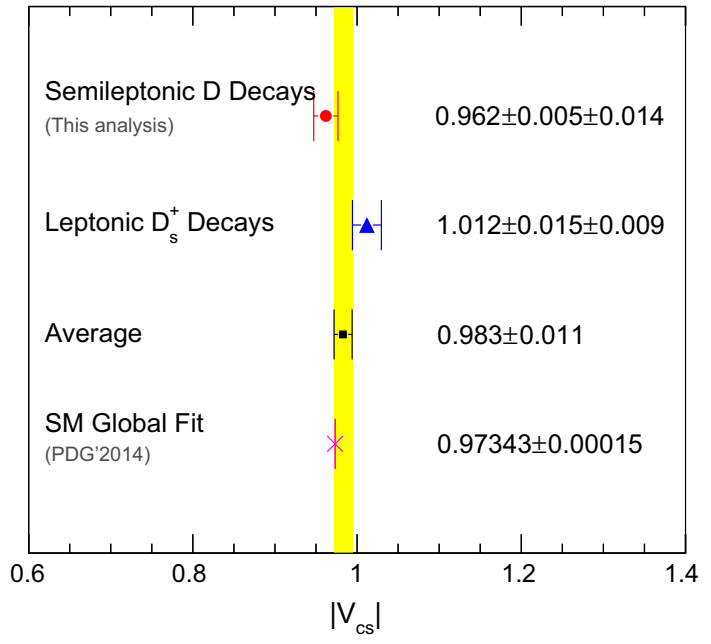

Fig. 5 Comparison of $\left|V_{c s}\right|$ extracted from semileptonic $D$ decays in this analysis with the one extracted from leptonic $D_{s}^{+}$decays and the one from SM global fit

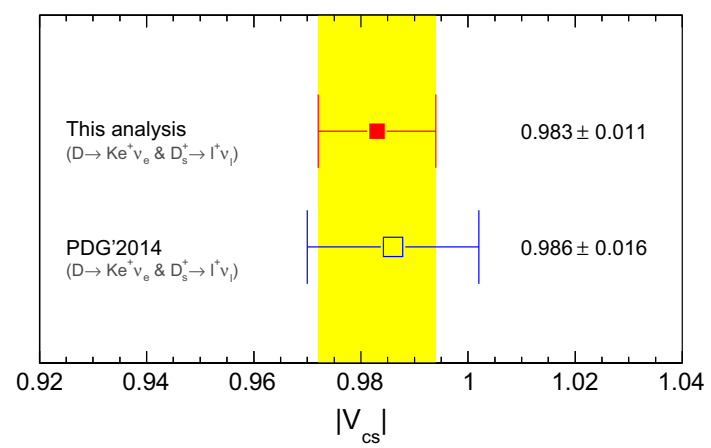

Fig. 6 Comparison of $\left|V_{c S}\right|$ extracted from semileptonic $D$ decays and leptonic $D_{s}^{+}$decays in this analysis with the PDG value

decays along with the PDG'2014 value of the $\left|V_{c s}\right|$ determined with CLEO-c, BaBar and Belle's measurements of $D \rightarrow K e^{+} v_{e}$ and $D_{s}^{+} \rightarrow \ell^{+} v_{\ell}$ decays [13].

The $\left|V_{c s}\right|^{D \rightarrow K e^{+} v_{e}}$ extracted from semileptonic $D$ decays deviates from the $\left|V_{c s}\right|_{s}^{D_{s}^{+} \rightarrow \ell^{+} v_{\ell}}$ extracted from leptonic $D_{s}^{+}$ decays by $2.2 \sigma$. This discrepancy may arise from three sources: (1) some new physic effects involved in leptonic $D_{s}^{+}$decays, which modify the decay rate; (2) underestimated decay constant $f_{D_{s}^{+}}$in LQCD; (3) overestimated form factor $f_{+}^{K}(0)$ in LQCD. Any of these would modify these decay rates resulting in shifts of the $\left|V_{c s}\right|^{D \rightarrow K e^{+} v_{e}}$ and $\left|V_{c s}\right|_{s}^{D_{s}^{+} \rightarrow \ell^{+} \nu_{\ell}}$, which are extracted from semileptonic $D$ and leptonic $D_{s}^{+}$decays, respectively.

\subsection{Effects of radiative correction on decay rate}

From experimental aspect, it's difficult to exclude the soft photon emission in the final state in the procedure of event selection. As a consequence, the experimentally measured branching fractions or partial decay rates usually include the contribution from the soft photon emission in the final states more or less. The theoretical prediction for the decay rate, Eq. (1), used in the extractions of form factor $f_{+}^{K}(0)$ and CKM matrix element $\left|V_{c s}\right|$ is for the tree-level semileptonic $D$ decay process. As the experimental precision of the decay rate measurement has already achieved an accuracy level of $0.5 \%$, in addition to improve the precision of the LQCD calculation for $f_{+}^{K}(0)$, it should be also necessary to take into account the radiative correction in further precise determination of $\left|V_{c s}\right|$. However, unlike the situation in $K_{\ell 3}$ decays, due to the lack of an universally valid effective theory, the theoretical or phenomenological estimation of radiative corrections in the semileptonic $D$ decays is absent at present stage. So we ignore this radiative correction in the analysis at present.

\section{Summary}

By globally analyzing all existing branching fractions of the $D \rightarrow K e^{+} v_{e}$ decays measured at earlier experiments and recent BESIII experiment as well as the precise measurements of partial decay rates in $q^{2}$ bins performed at the BaBar and CLEO-c experiments together, we obtain the most precise product of form factor and the magnitude of CKM matrix element $V_{c s}$ from a comprehensive $\chi^{2}$ fit. This obtained product reflects all of measurements for $D \rightarrow K e^{+} v_{e}$ decays in the world in the last 25 years. With the obtained $f_{+}^{K}(0)\left|V_{c s}\right|$ in conjunction with $\left|V_{c S}\right|$ from SM global fit, we determine the form factor

$f_{+}^{K}(0)=0.737 \pm 0.004 \pm 0.000$

which is in good agreement within error with LQCD calculations, but more precise than the most accurate LQCD calculation of the form factor by 2.8 factors. Alternately, with the recent most precise semileptonic $D \rightarrow K e^{+} v_{e}$ decay form factor calculated in LQCD, we obtain the $\left|V_{c s}\right|^{D \rightarrow K e^{+} v_{e}}=$ $0.962 \pm 0.005 \pm 0.014$, where the error is dominated by the uncertainties in LQCD calculation of the hadronic form factor. This determined $\left|V_{c s}\right|$ is in good agreement within error with the one from SM global fit, which indicates that no evidence of new physic effects involved in the semileptonic $D \rightarrow K e^{+} v_{e}$ decays is observed at present experimental accuracy level.

If combining the $\left|V_{c S}\right|^{D \rightarrow K e^{+} v_{e}}=0.962 \pm 0.005 \pm 0.014$ determined from semileptonic $D$ decays and $\left|V_{c s}\right|_{s}^{D_{s}^{+} \rightarrow \ell^{+} \nu_{\ell}}=$ $1.012 \pm 0.015 \pm 0.009$ determined from leptonic $D_{s}^{+}$decays together, we find

$\left|V_{c s}\right|=0.983 \pm 0.011$ 
which improves the accuracy of the PDG'2014 value $\left|V_{c S}\right|^{\mathrm{PDG}^{\prime} 2014}=0.986 \pm 0.016$ by $45 \%$, and is the most precisely extracted $\left|V_{c s}\right|$ from all existing measurements of semileptonic $D$ decays and leptonic $D_{s}^{+}$decays up to date.

Acknowledgments This work is supported in part by the Ministry of Science of Technology of China under Contracts No. 2009CB825204; National Natural Science Foundation of China (NSFC) under Contacts No. 10935007 and No. 11305180.

Open Access This article is distributed under the terms of the Creative Commons Attribution License which permits any use, distribution, and reproduction in any medium, provided the original author(s) and the source are credited.

Funded by $\mathrm{SCOAP}^{3}$ / License Version CC BY 4.0.

\section{Appendix A: Extraction of $\left|V_{c s}\right|$ from leptonic $D_{s}^{+}$ decays}

In this appendix, we present the determination of $\left|V_{c s}\right|$ by analyzing the existing measurements of leptonic $D_{s}^{+} \rightarrow$ $\ell^{+} \nu_{\ell}(\ell=\mu, \tau)$ decays.

In SM of particle physics, the decay width of $D_{s}^{+} \rightarrow \ell^{+} v_{\ell}$ is given by

$\Gamma\left(D_{s}^{+} \rightarrow \ell^{+} v_{\ell}\right)=\frac{G_{F}^{2}}{8 \pi} m_{\ell}^{2} m_{D_{s}^{+}}\left(1-\frac{m_{\ell}^{2}}{m_{D_{s}^{+}}^{2}}\right)^{2} f_{D_{s}^{+}}^{2}\left|V_{c s}\right|^{2}$,

where $m_{\ell}$ is the mass of lepton and $m_{D_{s}^{+}}$is the mass of $D_{s}^{+}$ meson. The parameter $f_{D_{s}^{+}}$is the decay constant, which is associated with the strong interaction effects between the two initial-state quarks.

In the past two decades, many measurements of leptonic $D_{s}^{+} \rightarrow \ell^{+} \nu_{\ell}$ decays were performed at $e^{+} e^{-}$experiments and fixed-target experiments. These measured branching fractions are summarized in Table 6.

To extract the magnitude of CKM matrix element $V_{c s}$, we globally analyze all of these existing measurements of leptonic $D_{s}^{+}$decay branching fractions shown in Table 6 . Assuming lepton universality, we construct a object function $\chi^{2}:$

$$
\begin{aligned}
\chi^{2}= & \sum_{i=1}^{5}\left(\frac{B_{\mu, i}^{\mathrm{ex}}-B_{\mu}^{\mathrm{th}}}{\sigma_{i}}\right)^{2}+\sum_{j=1}^{3}\left(\frac{R_{\mu, j}^{\mathrm{ex}}-R_{\mu}^{\mathrm{th}}}{\sigma_{j}}\right)^{2} \\
& +\sum_{k=1}^{6}\left(\frac{B_{\tau, k}^{\mathrm{ex}}-B_{\tau}^{\mathrm{th}}}{\sigma_{k}}\right)^{2},
\end{aligned}
$$

where $B_{\mu, i}^{\mathrm{ex}}$ is the $i$ th experimentally measured branching fraction of $D_{s}^{+} \rightarrow \mu^{+} v_{\mu}$ decay, $R_{\mu, j}^{\mathrm{ex}}$ is the $j$ th experimentally measured partial width of $D_{s}^{+} \rightarrow \mu^{+} v_{\mu}$ decay relative to the partial width of $D_{s}^{+} \rightarrow \phi \pi^{+}$decay, $B_{\tau, k}^{\text {ex }}$ is the $k$ th
Table 6 Measurements of $B\left(D_{s}^{+} \rightarrow \mu^{+} v_{\mu}\right)$ and $B\left(D_{s}^{+} \rightarrow \tau^{+} v_{\tau}\right)$

\begin{tabular}{ll}
\hline Experiment & $B\left(D_{s}^{+} \rightarrow \mu^{+} v_{\mu}\right)(\%)$ \\
\hline BES-I [31] & $1.5_{-0.6-0.2}^{+1.3+0.3}$ \\
ALEPH [32] & $0.68 \pm 0.11 \pm 0.18$ \\
CLEO-c [33] & $0.565 \pm 0.045 \pm 0.017$ \\
BaBar [34] & $0.602 \pm 0.038 \pm 0.034$ \\
Belle [35] & $0.531 \pm 0.028 \pm 0.020$ \\
Experiment & $\Gamma\left(D_{s}^{+} \rightarrow \mu^{+} \nu_{\mu}\right) / \Gamma\left(D_{s}^{+} \rightarrow \phi \pi^{+}\right)$ \\
BEATRICE [36] & $0.23 \pm 0.06 \pm 0.04$ \\
CLEO-II [37] & $0.173 \pm 0.023 \pm 0.035$ \\
BaBar [38] & $0.143 \pm 0.018 \pm 0.006$ \\
\hline Experiment & $B\left(D_{s}^{+} \rightarrow \tau^{+} \nu_{\tau}\right)(\%)$ \\
\hline L3 [39] & $7.4 \pm 2.8 \pm 2.4$ \\
OPAL [40] & $7.0 \pm 2.1 \pm 2.0$ \\
ALEPH [32] & $5.79 \pm 0.77 \pm 1.84$ \\
CLEO-c [41] & $5.58 \pm 0.33 \pm 0.13$ \\
BaBar [34] & $5.00 \pm 0.35 \pm 0.49$ \\
Belle [35] & $5.70 \pm 0.21_{-0.30}^{+0.31}$ \\
\hline
\end{tabular}

experimentally measured branching fraction of $D_{s}^{+} \rightarrow \tau^{+} \nu_{\tau}$ decay, and $\sigma$ denotes the combined statistical and systematic error of the measured (relative) branching fraction.

The theoretically predicted branching fraction of $D_{s}^{+} \rightarrow$ $\mu^{+} v_{\mu}$ is given by

$B_{\mu}^{\text {th }}=\Gamma\left(D_{s}^{+} \rightarrow \mu^{+} v_{\mu}\right) \times \tau_{D_{s}^{+}}$,

where $\Gamma\left(D_{s}^{+} \rightarrow \mu^{+} v_{\mu}\right)$ is calculated with Eq. (A1), and $\tau_{D_{s}^{+}}$ is the lifetime of $D_{s}^{+}$meson.

The theoretically expected ratio of $\Gamma\left(D_{s}^{+} \rightarrow \mu^{+} v_{\mu}\right)$ over $\Gamma\left(D_{s}^{+} \rightarrow \phi \pi^{+}\right)$is given by

$R_{\mu}^{\mathrm{th}}=B_{\mu}^{\mathrm{th}} / B\left(D_{s}^{+} \rightarrow \phi \pi^{+}\right)$,

where $B\left(D_{s}^{+} \rightarrow \phi \pi^{+}\right)$is the branching fraction of $D_{s}^{+} \rightarrow$ $\phi \pi^{+}$decay.

The theoretically predicted branching fraction of $D_{s}^{+} \rightarrow$ $\tau^{+} \nu_{\tau}$ is given by

$B_{\tau}^{\text {th }}=\Gamma\left(D_{s}^{+} \rightarrow \tau^{+} v_{\tau}\right) \times \tau_{D_{s}^{+}}$,

where $\Gamma\left(D_{s}^{+} \rightarrow \tau^{+} v_{\tau}\right)$ is calculated with Eq. (A1).

To obtain the experimentally measured product $f_{D_{s}^{+}}\left|V_{c s}\right|$, we perform a $\chi^{2}$ fit to these measured branching fractions for $D_{s}^{+} \rightarrow \ell^{+} \nu_{\ell}$ decays shown in Table 6. In the fit, we use $m_{\mu}=$ $(105.6583715 \pm 0.0000035) \mathrm{MeV} / c^{2}, m_{\tau}=(1776.82 \pm$ 0.16) $\mathrm{MeV} / c^{2}, m_{D_{s}^{+}}=(1968.30 \pm 0.11) \mathrm{MeV} / c^{2}, \tau_{D_{s}^{+}}=$ $(500 \pm 7) \times 10^{-15} \mathrm{~s}$, and $B\left(D_{s}^{+} \rightarrow \phi \pi^{+}\right)=(4.5 \pm 0.4) \%$ which are all quoted from PDG [13]. The product of the decay 
constant and the magnitude of CKM matrix element $V_{c s}$ is the only free parameter in the fit. The fit returns

$f_{D_{s}^{+}}\left|V_{c s}\right|=(252.0 \pm 3.7 \pm 1.8) \mathrm{MeV}$,

where the first error is from the statistical and systematic uncertainties in the measured (relative) branching fractions, and the second error is due to the uncertainties in the masses of lepton and $D_{s}^{+}$meson, the lifetime of $D_{s}^{+}$meson, and the branching fraction of $D_{s}^{+} \rightarrow \phi \pi^{+}$decay.

Dividing the product $f_{D_{s}^{+}}\left|V_{c s}\right|$ by the value $f_{D_{s}^{+}}=$ $\left(249.0 \pm 0.3_{-1.5}^{+1.1}\right) \mathrm{MeV}$ which is the newest and most precise value of decay constant calculated in LQCD with $N_{f}=$ $2+1+1$ quark flavors [42], we obtain

$\left|V_{c s}\right|^{D_{s}^{+} \rightarrow \ell^{+} v_{\ell}}=1.012 \pm 0.015 \pm 0.009$,

where the first error is from the statistical and systematic uncertainties in the measured (relative) branching fractions, and the second error is mainly due to the uncertainties in the lifetime of $D_{s}^{+}$meson, and the $f_{D_{s}^{+}}$calculated in LQCD.

Alternatively, by inserting $\left|V_{c s}\right|=0.97343 \pm 0.00015$ from the SM global fit [13] into Eq. (A6), we determine

$f_{D_{s}^{+}}=(258.9 \pm 3.8 \pm 1.8) \mathrm{MeV}$,

which is the most precisely determined $D_{s}^{+}$leptonic decay constant.

\section{References}

1. G. Rong, Chin. Phys. C 34, 788 (2010)

2. J.C. Anjos et al., The Tagged Photon Spectrometer Collaboration, Phys. Rev. Lett. 62, 1587 (1989)

3. G. Crawford et al., CLEO Collaboration, Phys. Rev. D 44, 3394 (1991)

4. A. Bean et al., CLEO Collaboration, Phys. Lett. B 317, 647 (1993)

5. B. Aubert et al., BaBar Collaboration, Phys. Rev. D 76, 052005 (2007)

6. B. Aubert et al., BaBar Collaboration, Phys. Rev. Lett. 100, 051802 (2008)

7. S. Dobbs et al., CLEO Collaboration, Phys. Rev. D 76, 112001 (2007)

8. M. Artuso et al., CLEO Collaboration, Phys. Rev. Lett. 80, 3193 (1998)

9. R. Barate et al., ALEPH Collaboration, Phys. Lett. B 403, 367 (1997)
10. D. Decamp et al., ALEPH Collaboration, Phys. Lett. B 266, 218 (1991)

11. H. Albrecht et al., ARGUS Collaboration, Phys. Lett. B 340, 125 (1994)

12. Q. He et al., CLEO Collaboration, Phys. Rev. Lett. 100, 091801 (2008)

13. K.A. Olive et al., Particle Data Group, Chin. Phys. C 38, 090001 (2014)

14. J.M. Link et al., FOCUS Collaboration, Phys. Lett. B 607, 233 (2005)

15. J. Adler et al., Mark III Collaboration, Phys. Rev. Lett. 62, 1821 (1989)

16. M. Ablikim et al., BES Collaboration, Phys. Lett. B 597, 39 (2004)

17. M. Ablikim et al., BES Collaboration, Phys. Lett. B 608, 24 (2005)

18. Y.H. Zheng, For BESIII Collaboration, ICHEP2014, 2-7 July 2014, Valencia, Spain

19. H.L. Ma, For BESIII Collaboration, Beauty 2014, 14-18 July 2014, Edinburgh, UK

20. G. Rong, For BESIII Collaboration, CKM2014, 8-12 September 2014, Vienna, Austria. arXiv:1411.3868 [hep-ex]

21. D. Besson et al., CLEO Collaboration, Phys. Rev. D 80, 032005 (2009)

22. L. Widhalm et al., Belle Collaboration, Phys. Rev. Lett. 97, 061804 (2006). arXiv:hep-ex/0604049

23. W.-M. Yao et al., J. Phys. G 33, 1 (2006)

24. D. Becirevcic, A.B. Kaidalov, Phys. Lett. B 478, 417 (2000)

25. D. Scora, N. Isgur, Phys. Rev. D 52, 2783 (1995)

26. T. Becher, R.J. Hill, Phys. Lett. B 633, 61 (2006)

27. J. Koponen et al., HPQCD Collaboration, arXiv:1305.1462 [heplat]

28. H. Na et al., HPQCD Collaboration. Phys. Rev. D 82, 114506 (2010)

29. C. Aubin et al., Fermilab Lattice Collaboration, MILC Collaboration, HPQCD Collaboration, Phys. Rev. Lett. 94, 011601 (2005)

30. A. Khodjamirian et al., Phys. Rev. D 80, 114005 (2009)

31. J.Z. Bai et al., BES Collaboration. Phys. Rev. Lett. 74, 4599 (1995)

32. A. Heister et al., ALEPH Collaboration. Phys. Lett. B 528, 1 (2002)

33. J.P. Alexander et al., CLEO Collaboration. Phys. Rev. D 79, 052001 (2009)

34. P. del Amo Sanchez et al., BaBar Collaboration, Phys. Rev. D 82, 091103(R) (2010)

35. A. Zupanc et al., Belle Collaboration, JHEP 09, 139 (2013)

36. Y. Alexandrov et al., BEATRICE Collaboration, Phys. Lett. B 478, $31(2000)$

37. M. Chada et al., CLEO Collaboration, Phys. Rev. D 58, 032002 (1998)

38. B. Aubert et al., BaBar Collaboration, Phys. Rev. Lett. 98, 141801 (2007)

39. M. Acciarri et al., L3 Collaboration, Phys. Lett. B 396, 327 (1997)

40. G. Abbiendi et al., OPAL Collaboration, Phys. Lett. B 516, 236 (2001)

41. P. Naik et al., CLEO Collaboration, Phys. Rev. D 80, 112004 (2009)

42. A. Bazavov et al., Fermilab Lattice and MILC Collaborations, Phys. Rev. D 90, 074509 (2014). arXiv:1407.3772 [hep-lat] 